\title{
Long term safety and efficacy of unilateral deep brain stimulation of the thalamus for parkinsonian tremor
}

\author{
K E Lyons, W C Koller, S B Wilkinson, R Pahwa
}

\begin{abstract}
The objective was to investigate the long term safety and efficacy of unilateral deep brain stimulation (DBS) of the VIM nucleus of the thalamus in Parkinson's disease.

Twelve patients with Parkinson's disease underwent unilateral DBS of the thalamus for medication resistant tremor between 1994 and 1997. Patients were evaluated with the motor section of the unified Parkinson's disease rating scale (UPDRS) in the medication on state at baseline, 3 months, 12 months, and yearly thereafter. Three patients were lost to follow up. Nine patients had follow up evaluations greater than 24 months and were included in the analyses. The last postsurgical follow up occurred on average 40.0 (SD 17.2) months after surgery. Tremor scores were significantly improved with stimulation on at the long term follow up compared with baseline. There was no significant change in UPDRS motor scores at long term follow up compared with baseline. There was no significant change in any stimulus parameters from 3 months to the long term follow up. Two patients had asymptomatic intracerebral haemorrhages and one patient had a subcutaneous haematoma over the implantable pulse generator site. Stimulus related adverse reactions were mild and easily controlled with changes in stimulus parameters. Two patients had replacement of the implantable pulse generator due to normal battery depletion, one patient had lead repositioning due to migration, and one patient had the lead extension wire replaced due to erosion.

In conclusion, unilateral DBS of the thalamus has long term efficacy for treatment of tremor due to Parkinson's disease.

(F Neurol Neurosurg Psychiatry 2001;71:682-684)
\end{abstract}

Keywords: Parkinson's disease; tremor; deep brain stimulation

Deep brain stimulation (DBS) of the thalamus is being increasingly used for the treatment of severe disabling tremor due to essential tremor. Although thalamic stimulation is also used as a treatment for tremor due to Parkinson's disease, a major concern has been a lack of functional improvement in patients with Parkinson's disease who have undergone this procedure. ${ }^{1}$ Deep brain stimulation of other nuclei such as the globus pallidus interna (GPi) and the subthalamic nucleus (STN) has improved tremor and provided functional improvement by reducing additional parkinsonian symptoms..$^{2-6}$ Many patients with Parkinson's disease have medication resistant tremor. ${ }^{7}$ In these patients, tremor can cause appreciable disability. These patients may not have bradykinesia or rigidity severe enough to recommend bilateral STN or GPi surgery; however, they may benefit from unilateral thalamic stimulation for tremor. Deep brain stimulation of the thalamus has been shown to dramatically reduce tremor due to Parkinson's disease; however, there are few studies that have evaluated the long term safety and efficacy of thalamic stimulation in Parkinson's disease.${ }^{18}$ Therefore, we have evaluated the long term effects of unilateral thalamic stimulation in this disease .

\section{Methods}

PATIENT SELECTION

Twelve patients with Parkinson's disease received unilateral DBS of the thalamus for medication resistant tremor at the University of Kansas Medical Center from 1994-7. The tremor had to cause significant disability despite pharmacological treatment. No patient had surgery outside of the study. Tremor had to be 3 or 4 in severity on a tremor rating scale of 0 to 4 in which 0 was equal to no tremor and 4 was severe tremor. All patients gave informed consent.

SURGICAL PROCEDURE AND PROGRAMMING

The procedure has been described in detail previously. ${ }^{1}$ Electrode model 3387 with an interelectrode distance of $1.5 \mathrm{~mm}$ was used for all patients. The implantable pulse generator model Itrel II was programmed to yield the greatest tremor suppression with the fewest side effects. Stimulation parameters and contact selection were programmed by telemetry, using a Medtronic Model 7432 console 
Table 1 UPDRS scores (medication on, stimulation on) and stimulation variable settings (mean (SD))

\begin{tabular}{llllll}
\hline & Baseline & 3 Months & 12 Months & $\begin{array}{l}\text { Long term } \\
\text { follow up }\end{array}$ & p Value \\
\hline UPDRS Tremor & $7.2(1.4)$ & $1.3(1.1)$ & $2.2(1.3)$ & $0.9(1.2)$ & 0.007 \\
UPDRS Motor & $40.7(13.9)$ & $29.2(7.2)$ & $32.4(12.7)$ & $35.6(11.2)$ & NS \\
Amplitude (V) & & $3.2(0.6)$ & $3.6(0.5)$ & $3.6(0.7)$ & NS \\
Pulse width ( $\mu$ s) & & $76.7(40.0)$ & $90.0(42.4)$ & $80.0(26.0)$ & NS \\
Rate (Hz) & $155.0(25.6)$ & $166.7(22.6)$ & $158.3(24.0)$ & NS \\
Polarity & & 9 Unipolar & 8 Unipolar & 3 Unipolar & \\
\hline
\end{tabular}

programmer and Model 745B MemoryMod software cartridge. Adjustable parameters included contact selection, pulse width, rate, and amplitude. Stimulation was initiated 1 day postoperatively unless the patient exhibited a microthalamotomy effect, defined as tremor reduction with the stimulator off and is assumed to be due to the trauma of electrode placement. Patients were instructed on how to switch their device on and off, using a hand held magnet, and told to turn their device off at night when possible to maximise battery life.

EVALUATIONS

The motor section (part III) of the unified Parkinson's disease rating scale (UPDRS) was performed with medications on at baseline and with medications on and stimulation on postsurgery at 3 months, 12 months and yearly thereafter.

\section{STATISTICAL ANALYSIS}

Wilcoxon signed rank comparisons for nonparametric data were used to compare UPDRS scores at baseline and follow up evaluations. Analysis of variance (ANOVA) was used to compare stimulation parameters across visits. Significance was set at $\mathrm{p}<0.05$.

\section{Results}

DEMOGRAPHICS

Twelve patients with Parkinson's disease underwent unilateral DBS of the VIM nucleus of the thalamus at the University of Kansas Medical Center for medication resistant tremor. Three patients were lost to follow up before 24 months and were not included in the analyses. One patient died 12 months after surgery from unrelated causes. Telephone interviews disclosed that one patient had maintained good tremor control; however, his other Parkinson's disease symptoms had progressed to a degree that rendered him immobile and unable to travel. The other patient declined to return after the 12 month visit and 2 years later he had his DBS system explanted due to loss of benefit and a pallidotomy was performed. Nine patients (eight men, one woman; average age 67.8 (SD 5.2) years; average disease duration 8.1 (SD 3.5) years) were included in the analyses. These patients were evaluated at an average postsurgical follow up of 40.0 (SD 17.2) months with a range of 26 to 66 months. Six patients had left brain implants and three patients had right brain implants.

\section{EVALUATIONS}

Motor scores of the UPDRS (part III) did not significantly change from baseline to long term follow up (table 1). However, tremor scores for the targeted side (questions 22 and 23 of the UPDRS) were significantly improved with stimulation on at long term follow up compared with baseline (table 1).

\section{STIMULUS PARAMETERS}

There was no significant change in any stimulus parameters from 3 months to last follow up at 40 months (table 1).

\section{ADVERSE EVENTS}

Surgical adverse events for the 12 patients included three patients with asymptomatic bleeds and one patient with a subcutaneous haematoma over the implantable pulse generator site. Stimulation adverse events included paraesthesia (12), headache (five), dysarthria (three), disequilibrium (three), and visual disturbances (two). These adverse events were mild and easy to manage with adjustments of the parameter settings. Device complications included lead reposition in two patients due to migration, lead extension wire replacement in one patient due to erosion, implantable pulse generator replacement in two patients due to normal battery depletion, implantable pulse generator and extension wire replacement in one patient due to shocking sensations, and full system explantation with subsequent pallidotomy in one patient due to loss of benefit. There was one unrelated death at 12 months (possible myocardial infarction during sleep) and two unrelated deaths (sepsis and respiratory arrest) after 24 months.

\section{Discussion}

Our study shows that thalamic stimulation is effective for the long term management of tremor in Parkinson's disease; however, other Parkinson's disease symptoms such as bradykinesia, rigidity, and gait and balance abnormality are not improved with thalamic stimulation. In our study, seven patients (58\%) indicated global improvement compared with baseline, one patient indicated no change $(8 \%)$, and three patients $(25 \%)$ reported good long term tremor control; however, due to the progression of their other parkinsonian symptoms, they have become almost completely disabled. Therefore, great caution should be taken in evaluating the patient's complete symptom profile before determining the most appropriate DBS target site. As a large percentage of patients with Parkinson's disease have medication resistant tremor, thalamic stimulation may be the surgery of choice for patients with tremor predominant Parkinson's disease with little evidence of other disabling parkinsonian signs.

Long term follow up studies of thalamic stimulation in Parkinson's disease have been minimal. Blond et al ${ }^{9}$ reported on 10 patients with Parkinson's disease with a mean follow up period of 19.4 months. In nine of the 10 patients tremor suppression was maintained and one patient required thalamotomy due to loss of tremor control. Akinesia was unchanged in their patients and the authors thought that it was difficult to evaluate rigidity due to the severe tremor. Side effects were minimal. 
Albanese et $a l^{10}$ described 27 patients with Parkinson's disease who received thalamic stimulation. Six of their patients had bilateral implantation and 21 had unilateral implantation. The mean follow up was 0.9 years. Tremor scores improved by $73 \%$ in the upper limbs and $62 \%$ in the lower limbs. A slight reduction in rigidity was reported in three patients and moderate relief of bradykinesia in three patients. Complications included one intracranial haemorrhage, one skin erosion, one electrode breakage, two local infections, two lead replacements, and three catheter disconnections. Similarly, Hariz et al ${ }^{11}$ reported 22 patients with Parkinson's disease who received DBS of the VIM nucleus of the thalamus with a mean follow up of 21 months. There was a significant improvement in the motor section of the UPDRS and particularly on the tremor items of the UPDRS. Kumar et $a l^{12}$ reported on 11 patients with Parkinson's disease with a mean follow up of 16.2 months who had significant improvements in the contralateral arm and leg rest tremor but no overall improvement on the motor portion of the UDPRS or other symptoms of Parkinson's disease .

In our study, after an average of 40 months, there were no significant changes in stimulus parameters compared with the 3 month visit. Similarly, Albanese et $a l^{10}$ reported some change in stimulation parameters during the first 2 to 3 months after surgery but they did not report any changes over long term follow up. Hariz et $a l^{11}$ showed significant increases in stimulation parameters for up to 1 year; however, after 1 year of stimulation parameters seemed to stabilise. By contrast, Kumar et $a l^{12}$ reported that is was necessary to increase the current intensity over time to control tremor. These inconsistent findings related to changes in stimulus parameters could be related to variability in disease progression, the development of tolerance, or individual differences in initial programming parameters used.
We conclude that for patients with tremor predominant Parkinson's disease, thalamic deep brain stimulation provides long term clinical benefit for tremor. Deep brain stimulation of the thalamus was found to be a relatively safe procedure for the treatment of parkinsonian tremor. Surgical induced and stimulation induced adverse effects were mild and were quickly resolved. However, there were many device complications. Forty per cent (5/12) of our patients required additional surgical procedures to maintain good tremor control.

This work was supported in part by the Parkinson's Association of Greater Kansas City.

1 Koller W, Pahwa R, Busenbark K, et al. High-frequency unilateral thalamic stimulation in the treatment of essential and parkinsonian tremor. Ann Neurol 1997;42:292-9.

2 Pahwa R, Wilkinson S, Smith D, et al. High-frequency Pahwa R, Wilkinson $\mathrm{S}$, Smith $\mathrm{D}$, et al. High-frequency
stimulation of the globus pallidus for the treatment of Parstimulation of the globus pallidus for the treatm
kinson's disease. Neurology 1997;49:249-53.

3 Krack P, Pollak P, Limousin P, et al. Subthalamic nucleus or internal pallidal stimulation in young onset Parkinson's disease. Brain 1998;121:451-7.

4 Krack P, Benazzouz A, Pollak P, et al. Treatment of tremor in Parkinson's disease by subthalamic nucleus stimulation. Mov Disord 1998;13:907-14.

5 Kumar R, Lozano AM, Kim YJ, et al. Double-blind evaluation of subthalamic nucleus deep brain stimulation in advanced Parkinson's disease. Neurology 1998;51:850-5.

6 Kumar R, Lozano AM, Montgomery E, et al. Pallidotomy and deep brain stimulation of the pallidum and subthalamic nucleus in advanced Parkinson's disease. Mov Disord 1998;13(suppl 1):73-82.

7 Koller WC. Initiating treatment of Parkinson's disease. Neurology 1992;42(suppl 1):33-8.

8 Benabid AL, Pollak P, Gao D, et al. Chronic electrical stimulation of the ventralis intermedius nucleus of the thalamus as a treatment of movement disorders. 7 Neurosurg 1996;84:203-14.

9 Blond S, Caparrros-Lefebvre D, Parker F, et al. Control of tremor and involuntary movement disorders by chronic stereotactic stimulation of the ventral intermediate thalamic nucleus. $\mathcal{F}$ Neurosurg 1992;77:62-8.

10 Albanese A, Nordera GP, Caraceni T, et al. Longterm ventralis intermedius thalamic stimulation for parkinsonian tremor. Italian Registry for Neuromodulation in Movement Disorders. Adv Neurol 1999;80:631-4.

11 Hariz MI, Shamsgovara P, Johansson F, et al. Tolerance and tremor rebound following long-term chronic thalamic stimulation for parkinsonian and essential tremor. Stereotact Funct Neurosurg 1999;72:208-18.

12 Kumar K, Kelly M, Toth C. Deep brain stimulation of the ventral intermediate nucleus of the thalamus for control of tremors in Parkinson's disease and essential tremor. Stereotact Funct Neurosurg 1999;72:47-61. 